\title{
Two-player envy-free multi-cake division ${ }^{\star}$
}

\author{
John Cloutier ${ }^{\text {a }}$, Kathryn L. Nyman ${ }^{\text {b,* }}$, Francis Edward Su ${ }^{\mathrm{c}}$

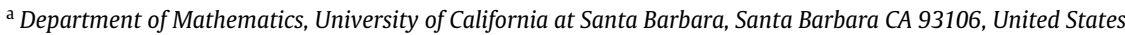 \\ ${ }^{\mathrm{b}}$ Department of Mathematics, Willamette University, Salem, OR 97301, United States \\ ${ }^{\mathrm{c}}$ Department of Mathematics, Harvey Mudd College, Claremont, CA 91711, United States
}

\section{A R T I C L E I N F O}

\section{Article history:}

Received 4 November 2008

Received in revised form

28 August 2009

Accepted 11 September 2009

Available online 16 September 2009

\section{JEL classification:}

C62

D63

D74

MSC:

52B05

$91 \mathrm{~B} 32$

Keywords:

Fair division

Envy-free

Sperner's lemma

Polytope

Labelings

\section{A B S T R A C T}

We introduce a generalized cake-cutting problem in which we seek to divide multiple cakes so that two players may get their mostpreferred piece selections: a choice of one piece from each cake, allowing for the possibility of linked preferences over the cakes. For two players, we show that disjoint envy-free piece selections may not exist for two cakes cut into two pieces each, and they may not exist for three cakes cut into three pieces each. However, there do exist such divisions for two cakes cut into three pieces each, and for three cakes cut into four pieces each. The resulting allocations of pieces to players are Pareto-optimal with respect to the division. We use a generalization of Sperner's lemma on the polytope of divisions to locate solutions to our generalized cakecutting problem.

(c) 2009 Elsevier B.V. All rights reserved.

\section{Introduction}

The classical cake-cutting problem of Steinhaus (1948) asks how to cut a cake fairly among several players, that is, how to divide the cake and assign pieces to players in such a way that each person gets what he or she believes to be a fair share. The word cake refers to any divisible good, and the word

\footnotetext{
This research was partially supported by NSF Grant DMS-0701308.

* Corresponding author. Tel.: +1503 37068886 .

E-mail addresses: john@math.ucsb.edu (J. Cloutier), knyman@willamette.edu (K.L. Nyman), su@math.hmc.edu (F.E.Su).
} 

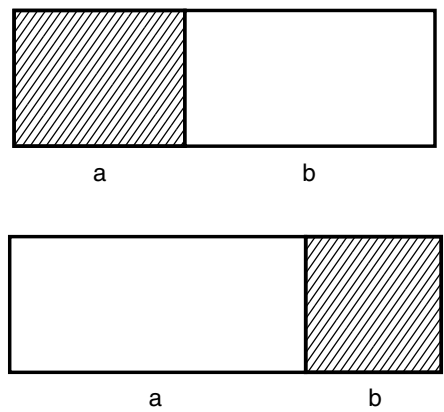

Fig. 1. The shaded and unshaded pieces represent disjoint piece selections $a b$ and $b a$, respectively.

fair can be interpreted in many ways. A strong notion of a fair division is an envy-free division, one in which every player believes that their share is better than any other share. Existence of envy-free cake divisions dates back to Neyman (1946), but constructive solutions are harder to come by; the recent procedure of Brams and Taylor (1995) was the first $n$-person envy-free solution. See Brams and Taylor (1996) and Robertson and Webb (1998) for surveys.

It is natural to consider the cake-cutting question when there is more than one cake to divide. Given several cakes, does an envy-free division of the cakes exist, and under what conditions can such a division be guaranteed?

Of course, if the player preferences over each cake are independent (i.e., additively separable), then the problem can be solved by one-cake division methods- simply perform an envy-free division on each cake separately. So the question only becomes interesting when the players have linked preferences over the cakes, in which the portion of one cake that a player prefers is influenced by the portion of another cake that she might also obtain.

Consider the case of two players, Alice and Bob, dividing two cakes. Suppose that each cake is to be cut into two pieces by a single cut, and players may choose one piece from each cake (called a piece selection). Note that there are 4 possible piece selections, only 2 of which will be chosen by Alice and Bob. See Fig. 1.

We would like the 2 chosen piece selections to be disjoint, i.e., have no common piece on either cake, if Bob and Alice are to avoid a fight. Also, we would like their piece selections to be envy-free, which means that each player would not want to trade their piece selection for any of other 3 possible piece selections. We remark that this notion of envy-free piece selection is stronger than the notion of an envy-free allocation, in which after pieces are allocated to players, a given player is only comparing their piece selection to what other players actually receive (in this example, there is just 1 other piece selection that has been assigned to a player). Our stronger notion of envy-freeness for piece selections ensures that the allocation is Pareto-optimal over all possible piece selections in that division.

Does there always exist a division of the cakes (by single cuts) so that the players have disjoint envy-free piece selections?

Consider the following scenario as an example of this generalized cake-cutting question. A company employs two part-time employees Alice and Bob who work half-days (either a morning or an afternoon shift) two days each week. Between them, they should cover the entire day on each of those two days. Now, Alice and Bob may have certain preferences (such as preferring a morning or afternoon shift) and such preferences may be linked (e.g., Alice might highly prefer the same schedule both days, whereas Bob might prefer to have a morning shift on one day if he has the afternoon on another).

Their boss would like to account for both of their preferences. Suppose that she has a certain fixed amount of salary money per day to divide up between the morning and afternoon shifts. Is there always a way to split the daily salary pool among the shifts so that Alice and Bob will choose different shifts? If so, is there a method to find it? If not, why not? Here, the cakes are the salary pools for each day, and the pieces of cake are the shift assignments along with their salary. 
In this paper we examine what can be said about envy-free assignments in situations like this one. In particular, we show that for two cakes, single cuts on each cake are insufficient to guarantee an envy-free allocation of piece selections to players; there exist preferences that Alice and Bob could have for which it is impossible to cut the cakes so that they would prefer disjoint piece selections. However, if one of those two cakes is divided into 3 pieces, then, somewhat surprisingly, there does exist an envy-free allocation of piece selections to players (with one unassigned piece).

Similarly, for three or more cakes, cutting each cake into three pieces is not sufficient to guarantee the existence of an envy-free allocation of piece selections to two players. However, if each cake is cut into four pieces, we find that such an allocation is always possible. These results are reminiscent of the cake-cutting procedure of Brams and Taylor (1995) in which the authors show that for more than two players, the number of pieces required to ensure an envy-free division is strictly larger than the number of players. Here, however, we find that extra pieces are necessary even in the two-player case.

The key idea here is to view the space of possible divisions as a polytope; we triangulate this polytope and label each vertex in a way that reflects player preferences for piece selections in the division that the vertex represents. The labels satisfy the conditions of a generalization of Sperner's lemma (Sperner, 1928) to polytopes; its conclusion suggests a division and a disjoint allocation of piece selections to players that is envy-free, and Pareto-optimal with respect to all possible piece selections in that division.

\section{The polytope of divisions and the polytopal Sperner lemma}

Sperner's lemma (Sperner, 1928) is a combinatorial analogue of the Brouwer fixed point theorem, one of the most important theorems of mathematics and economics. Constructive proofs of Sperner's lemma can be used to prove the Brouwer theorem in a constructive fashion; such proofs have therefore found wide application in game theory and mathematical economics to locate fixed points as well as Nash equilibria (e.g., see Todd (1976) and Yang (1999) for surveys).

More recently, Sperner's lemma and related combinatorial theorems have been used to show the existence of envy-free divisions for a variety of fair division problems, including the classical cakecutting question, e.g., see Su (1999) and Simmons and Su (2003). In this paper we will use a recent generalization of Sperner's lemma to polytopes (De Loera et al., 2002) to address our generalized cakecutting question.

Before describing Sperner's lemma or its generalization, we review some terminology. A d-simplex is a generalization of a triangle or tetrahedron to $d$ dimensions: it is the convex hull of $d+1$ affinely independent points in $\mathbb{R}^{d}$. A polytope $P$ in $\mathbb{R}^{d}$ is the convex hull of $n$ points $v_{1}, v_{2}, \ldots, v_{n}$, called the vertices of the polytope. We call an $n$-vertex, $d$-dimensional polytope an $(n, d)$-polytope. A face of a polytope is the convex hull of any subset of the vertices of that polytope; a $(d-1)$-dimensional face of $P$ is called a facet.

A triangulation $T$ of $P$ is a finite collection of distinct simplices such that: (i) the union of all the simplices in $T$ is $P$, (ii) the intersection of any two simplices in $T$ is either empty or a face common to both simplices and (iii) every face of a simplex in $T$ is also in $T$. The vertices of simplices in $T$ are called vertices of the triangulation $T$.

A Sperner labeling of $T$ is a labeling of the vertices of $T$ that satisfies these conditions: (1) all vertices of $P$ have distinct labels and (2) the label of any vertex of $T$ which lies on a facet of $P$ matches the label of one of the vertices of $P$ that spans that facet. A full cell is any $d$-dimensional simplex in $T$ that is fullylabeled: each of its $d+1$ vertices possess distinct labels. See Fig. 2 for examples of Sperner-labeled polygons (with full cells shaded).

Sperner's lemma (Sperner, 1928) states that any Sperner-labeled triangulation of a simplex contains an odd number of full cells. The generalization which will be of use to us is:

Theorem 1 (De Loera-Peterson-Su). Any Sperner-labeled triangulation of an $(n, d)$-polytope $P$ must contain at least $(n-d)$ full cells.

For instance, the Sperner-labeled pentagon in Fig. 2 has at least $5-2=3$ full cells. 

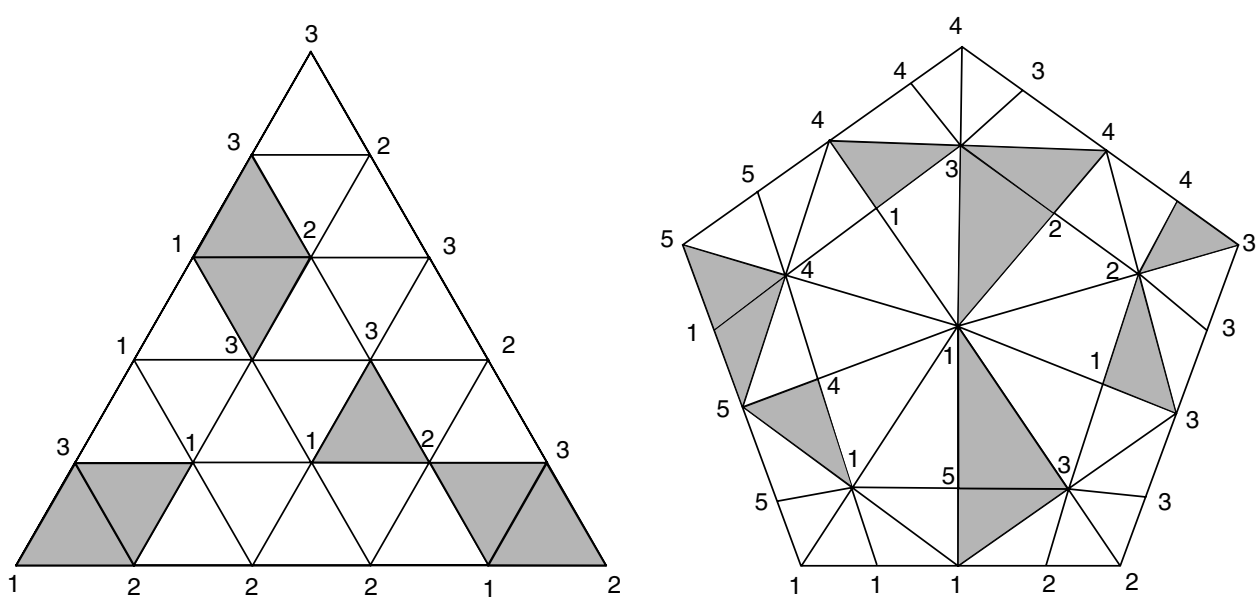

Fig. 2. A Sperner-labeled triangle (2-simplex) and pentagon ((5, 2)-polytope). Full cells are shaded.

The polytope that will interest us is the polytope of divisions, which we describe presently. Suppose that we have $m$ cakes, each of length 1 , and that each cake is to be cut into $k$ pieces. Let the length of the $j$ th piece of the $i$ th cake be denoted by $x_{i j}$. Then for all $1 \leq i \leq m$ we have

$$
x_{i 1}+x_{i 2}+\cdots+x_{i k}=1 .
$$

Each such division can be represented by an $m \times k$ matrix:

$$
\left(\begin{array}{cccc}
x_{11} & x_{12} & \ldots & x_{1 k} \\
x_{21} & x_{22} & \ldots & x_{2 k} \\
\vdots & \vdots & \ddots & \vdots \\
x_{m 1} & x_{m 2} & \ldots & x_{m k}
\end{array}\right)
$$

where each row sum is 1 .

Now, suppose that each cake is cut in such a way that one piece is the entire cake and all other pieces are empty. We will call such a "division" of the cakes a pure division. The matrix representation of a pure division is one in which each row has exactly one entry as a 1 and the rest 0 . Notice that any division may be written as a convex linear combination of the pure divisions. Thus, it is natural to view the space of divisions as a polytope $P$ with the pure divisions as its vertices. We call $P$ the polytope of divisions. From the matrix representation of this polytope we see that the space of divisions is of dimension $m(k-1)$, since in each of the $m$ cakes, the length of the last piece is determined by the lengths of the first $k-1$ pieces. Also $P$ has $k^{m}$ vertices, one for each pure division. Thus $P$ is an $(n, d)$ polytope with $d=m(k-1)$ and $n=k^{m}$.

\section{The owner labeling and the preference labeling}

We now describe how to locate envy-free piece selections using the polytope of divisions $P$. Suppose that there are two players, $A$ and $B$. Let $T$ be a triangulation of $P$. Next, label $T$ with an owner labeling: label each vertex in $T$ with either $A$ or $B$. We will want this owner labeling to satisfy the condition that each simplex in $T$ has roughly the same number of $A$ and $B$ labels.

In general, for any number of players, call an owner labeling uniform if in each simplex, the number of labels for each player differs by at most one from any other player. So for two players $A$ and $B$, if a simplex $\sigma$ in $T$ has $n$ vertices, a uniform labeling would assign labels $A$ and $B$ to $n / 2$ vertices each if $n$ is even, and to at least $(n-1) / 2$ vertices each if $n$ is odd.

We claim that any polytope has a triangulation of arbitrarily small mesh size that can be given a uniform owner labeling. In particular, this may be accomplished by choosing a triangulation $T$ of the 


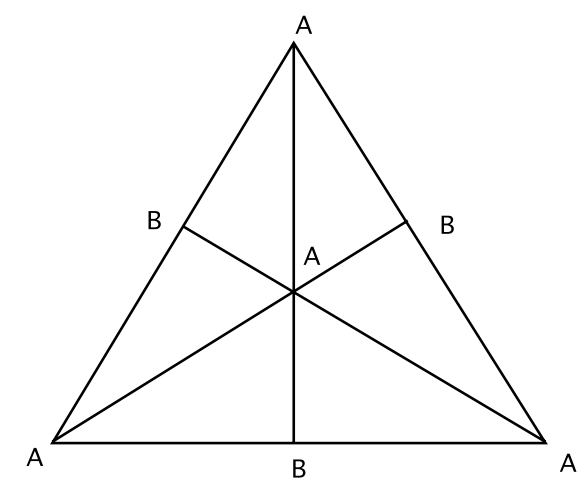

Fig. 3. A barycentric subdivision with uniform owner labeling for players $A$ and $B$.

required mesh size, and barycentrically subdividing $T$. A barycentric subdivision takes a $k \operatorname{simplex} \sigma$ of $T$, and replaces it by $k$ ! smaller $k$-simplices whose vertices are the barycenters of an increasing saturated chain of faces of $\sigma$. Since each smaller $k$-simplex contains exactly one barycenter in each dimension, we may assign the even-dimensional barycenters to player $A$ and the odd-dimensional barycenters to $B$. This owner labeling is uniform for players $A$ and $B$. See Fig. 3 for an example of a barycentric subdivision with a uniform owner labeling. (If there are more than two players, then cyclically rotate player assignments by dimension).

Now that the ownership has been assigned to the vertices of $T$, we shall construct a preference labeling of $T$.

Given a division (not necessarily pure) of the $m$ cakes, a piece selection is a choice of one piece from each cake. We say that a player prefers a certain piece selection if that player does not think that any other piece selection is better. We make the following three assumptions about preferences:

(1) Independence of preferences. A player's preferences depend only on that player and not on choices made by other players.

(2) The players are hungry. A player will always prefer a nonempty piece in each cake to an empty piece in that cake (assuming the pieces selected in the other cakes are fixed). Hence a preferred piece selection will contain no empty pieces.

(3) Preference sets are closed. If a piece selection is preferred for a convergent sequence of divisions, then that piece selection will be preferred in the limiting division.

Note that with these assumptions about player preferences, a given player always prefers at least one piece selection in any division and may prefer more than one if that player is indifferent between piece selections.

We now construct the preference labeling of $T$. Notice that a vertex $v$ of $T$ is just a point in $P$ and so it represents a division of the cakes. Ask the owner of $v$ (who is either $A$ or $B$ ):

"which piece selection do you prefer in this division?"

Label $v$ by the answer given. Since every piece selection corresponds to a pure division in a natural way (namely, each may be thought of as a choice of one piece from each cake), we obtain a preference labeling of the vertices of $T$ by pure divisions.

This new labeling is, in fact, a Sperner labeling. To see why, note that each vertex of $P$ is a pure division, and condition (2) ensures that the player who owns that vertex will select the unique nonempty piece from each cake. So, every vertex of $P$ will be labeled by its corresponding pure division. A vertex $v$ of $T$ that lies on a facet of $P$ is a strict convex linear combination of the subset of vertices of $P$ that span that facet. Thus, the division represented by $v$ is a strict convex linear combination of the subset of pure divisions that are represented by the vertices that span the facet. So, if the ith piece (on any cake) of the division represented by $v$ is empty, then each of those pure divisions must have an empty ith piece as well. So condition (2) guarantees that the owner of $v$ will never prefer a piece selection that corresponds to a pure division that is not on the same facet as $v$. Thus, the preference labeling is a Sperner labeling. 
The polytopal Sperner lemma shows that there exist $(n-d) d$-dimensional, full cells in $T$. The owner labeling of each of these cells is uniform. Hence, a full cell represents $d+1$ similar divisions in which players $A$ and $B$ choose different piece selections.

Now, if we repeat this procedure for a sequence of finer and finer triangulations of $P$, we would create a sequence of smaller and smaller full cells. Since $P$ is compact, there must be a convergent subsequence of full cells that converges to a single point. Since each full cell in the convergent subsequence also has a uniform owner labeling and since there are only finitely many ways to choose a piece selection, there must be an infinite subsequence of our convergent sequence for which the piece selections of each player remain unchanged. Condition (3) guarantees that the selections will not change at the limit point of these full cells. So, at this limit point, the players choose different piece selections just as they did in the cells of the sequence.

In this way, we may find a division for which both players choose different piece selections, that is, selections where the players choose different pieces on at least one cake. More generally, with any number of players, the same arguments (using a uniform labeling over multiple players) show:

Theorem 2. Given p players and $m$ cakes, if each cake is cut into $k$ pieces each and $k \geq 1+(p-1) / m$, then there exists a division of the cakes for which each player chooses different (though not necessarily disjoint) piece selections.

In particular, this theorem holds if each cake is cut into $k=p$ pieces. The bound on the number of pieces comes from the fact that the number of vertices of the simplex must be at least as large as the number of players so that each player owns at least one vertex of the full cell. The conclusion says that the piece selections chosen are envy-free: no one would trade their piece selection for any other. However, this theorem does not help us allocate the pieces, because two different players may have conflicting piece selections if they chose the same piece on a particular cake.

So we wish to find divisions for which the players make disjoint envy-free piece selections, i.e., selections where the players would choose non-conflicting pieces on every cake. We remark that such an allocation would be Pareto-optimal with respect to all possible piece selections in that division. This is because each player would have chosen, over all piece selections, what he/she most prefers. So there would be no other allocation of pieces of a given division in which players could do better.

We explain this in contrast to an interesting example of Brams et al. (2001), who consider a single cake cut into 6 pieces in which 3 players choose 2 pieces each. They exhibit a division and allocation of a pair of pieces to each player in such a way that the allocation is envy-free but not Pareto-optimal, because for the given 6-piece division there is another allocation in which some players are better off. Although theirs was an envy-free allocation (involving comparisons of 3 allocated pairs of pieces), those pairs were not envy-free piece selections (involving comparisons of 30 possible pairs of pieces). A division into envy-free piece selections would by nature yield a Pareto-optimal allocation with respect to the given division.

In what follows we explore the existence of disjoint envy-free piece selections.

\section{Divisions of two cakes}

\subsection{Two cakes, two pieces each}

We now explore what happens when two cakes, each cut into two pieces, are divided among two players: $A$ and $B$. We show:

Theorem 3. Given 2 players and 2 cakes, there does not necessarily exist a division of the cakes into 2 pieces each that contains disjoint envy-free piece selections for those players.

Proof. Let $P$ be the polytope of divisions. In this case, $P$ is a $(4,2)$-polytope, i.e., a square. We label the pure divisions as follows: $a a, a b, b b, b a$, where, for instance, $a a$ represents the pure division in which the left pieces of Fig. 1 are the entire cakes. 


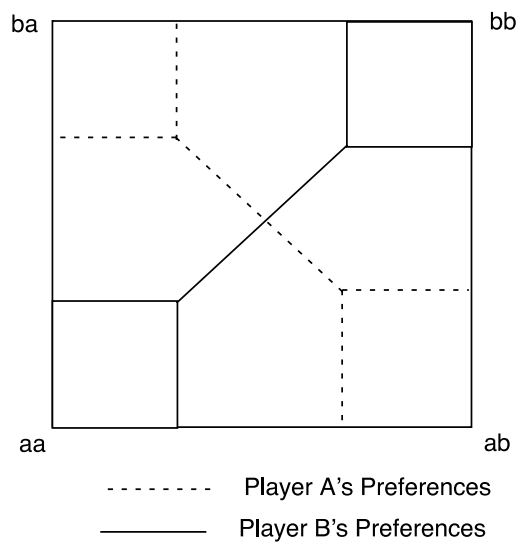

Fig. 4. Conflicting preferences for 2 players, 2 cakes and 2 pieces per cake.

Recall that the same names $a a, a b, b a, b b$ can be used to reference piece selections. Also note that $a a$ and $b b$ represent disjoint piece selections, and so do $a b$ and $b a$.

Player A's preferences can be described by a cover of the square $P$ by four sets, labeled by piece selections: $a a, a b, b a, b b$. This is accomplished by placing point $p$ in $P$ in the set $x y$ if in the division of cake represented by $p$, player $A$ would prefer piece selection $x y$. Some points may be in multiple sets (representing indifference between piece selections). Assumption (3) about preferences (see previous section) ensures that the four sets are closed. The union of the four sets contains $P$. Assumption (2) about preferences ensures that the pure divisions are in the sets that bear their own name.

In a similar fashion, player $B$ also has a cover by four sets that describes his preferences. To find a division that allows for disjoint envy-free piece selections, we seek a point $p$ in $P$ such that $p$ is in one player's $a a$ set and the other's $b b$ set, or in one player's $a b$ set and the other's $b a$ set.

Now consider the player preferences shown in Fig. 4, where we assume that each set is labeled by the pure division that it contains. A visual inspection shows that there is no such point $p$ as described above.

Therefore, it is not necessarily possible to divide two cakes into two pieces in such a way that the two players will choose different pieces on each cake, i.e., find disjoint piece selections. This result may seem somewhat unexpected, since if preferences are not linked, we can divide each individual cake in such a way that both players would be satisfied with their piece on each individual cake.

An interpretation of Fig. 4 provides an example of linked preferences. Player $A$ generally seems to either want the left hand or the right hand pieces of both cakes, and player $B$ generally wants both a left and a right hand piece (in both cases, as long as the pieces are not too small). In our running example from the introduction, the preferences in Fig. 4 would correspond to Alice strongly desiring to work either both morning or both afternoon shifts, and Bob strongly desiring to work one morning and one afternoon shift. There may then be no division of the salary pool on each day that will induce them to take disjoint shifts.

One may ask why the arguments of the previous section do not extend here. The answer is that while in each triangulation we can ensure an $A-B$ edge (in owner labels) that corresponds to endpoints with different preference labels, we cannot ensure that the corresponding piece selections are disjoint. Indeed we see from Fig. 4 that if $P$ is triangulated very finely, there will be no $A-B$ edge (in owner labels) that could possibly be $a a-b b$ or $a b$ - $b a$ (in preference labels) since the corresponding players' sets in the figure are not close.

In contrast to Theorem 3, we may obtain a positive result for two players when there are three players involved.

Theorem 4. Given 3 players and 2 cakes, there exists a division of the cakes, each cut into 2 pieces, such that some pair of players has disjoint envy-free piece selections. 


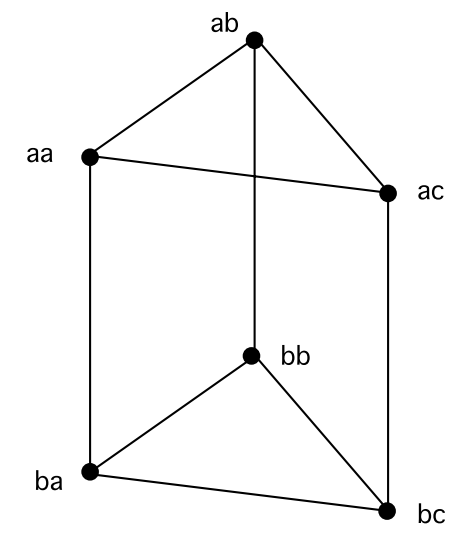

Fig. 5. The polytope of divisions for 2 cakes divided into 2 and 3 pieces.

Proof. With three players, consider an owner labeling in which every triangle has vertices labeled by $A, B$, and $C$, so that each vertex of the triangle belongs to a different player. Note that a fully-labeled triangle in the square of all divisions must contain a pair of disjoint piece selections. So, there is a pair of players who prefer disjoint piece selections for two very close divisions. Using the limiting argument of Section 3, there is a division in which some pair of players has disjoint envy-free piece selections.

\subsection{Envy-free piece selections with more pieces}

While there does not necessarily exist an envy-free allocation for two players with each cake cut into two pieces, we can satisfy both players with one cake cut into three pieces and the other cut in (only) two pieces. The proof of this fact, and later results for three cakes, will use the following theorem:

Theorem 5 (De Loera-Peterson-Su). Let $P$ be an $(n, d)$-polytope with Sperner-labeled triangulation $T$. Let $f: P \rightarrow P$ be the piecewise-linear map that takes each vertex of $T$ to the vertex of $P$ that shares the same label, and is linear on each d-simplex of T. The map $f$ is surjective, and thus the collection of full cells in $T$ forms a cover of $P$ under $f$.

Theorem 6. Given 2 players and 2 cakes, there is a division of the cakes- one cut into 2 (or more) pieces, the other cut into 3 (or more) pieces- so that the players have disjoint envy-free piece selections.

Proof. Let $P$ be the (6,3)-polytope of divisions of the two cakes. In this case, $P$ is a triangular prism with vertices corresponding to the pure divisions $a a, a b, a c, b a, b b$, and $b c$. Notice the 1-skeleton of $P$ can be interpreted as a graph in which piece selections that conflict appear as labels on adjacent vertices of $P$ (see Fig. 5).

Let $T$ be a triangulation of $P$ with a uniform owner labeling. By Theorems 1 and 5 , there exists a fully-labeled 3-simplex $\sigma$ whose image $f(\sigma)$, under the map $f$ of Theorem 5 , is one of the simplices of the cover of $P$. Therefore, $f(\sigma)$ is non-degenerate and its four vertices do not lie on a common face of $P$. In this case, one can verify that one vertex $v$ of $f(\sigma)$ must be non-adjacent, in the 1-skeleton of $P$, to two other vertices, $w$ and $y$. This means $w$ and $y$ correspond to piece selections that are disjoint from the piece selection of $v$. Without loss of generality, if $A$ owns $v, B$ must own at least one of $w$ and $y$ because the owner labeling is uniform. Therefore, using the methods of Section 3, we can find an envy-free allocation.

If the two cakes are divided into more than 2 or 3 pieces respectively, we can find an envy-free allocation simply by restricting our attention to divisions of the cake in which the extra pieces are empty, and using the above results. (But other envy-free divisions may exist as well.) 


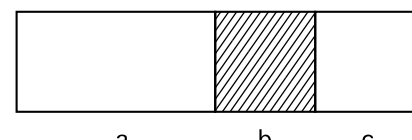

a $\quad$ b

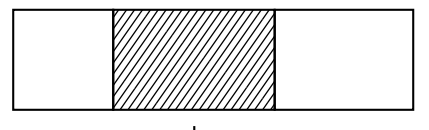

a b c

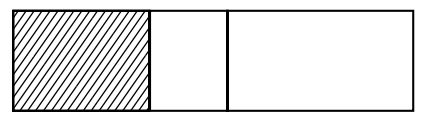

a $\quad b$

C

Fig. 6. A piece selection with two pieces of the same type ( $b b a)$.

\section{Three cakes}

\subsection{Three cakes, three pieces each}

As shown in Section 4.1, dividing two cakes into two pieces each was insufficient to guarantee a division with envy-free piece selections for two players. We shall see that for two players, dividing three or more cakes into three pieces each is also insufficient, but cutting three cakes into four pieces each guarantees a division with envy-free piece selections. We begin by examining divisions of three cakes into three pieces each.

Theorem 7. Given 2 players and 3 (or more) cakes, there does not necessarily exist a division of the cakes into 3 pieces each that contains disjoint envy-free piece selections for the two players.

Proof. We will let $a, b$, and $c$ designate the leftmost, center, and rightmost piece of each cake, such that a choice of $b b a$, for example, will refer to a player choosing the second piece in cakes one and two, and the first piece in cake three (see Fig. 6).

We now construct preferences for two players, Alice $(A)$ and Bob $(B)$, for which there does not exist a division with envy-free disjoint piece selections. Fix some small $\epsilon>0$. Let $A$ prefer piece selections according to the following broad categories in descending order of preference:

(1) three pieces of the same type (i.e., $a a a, b b b, c c c$ ),

(2) two pieces of the same type (e.g., $a b a, c c b$ ),

(3) three pieces all of different type (e.g., $a b c)$.

Let $B$ 's preferences be the reverse:

(1) three pieces all of different type,

(2) two pieces of the same type,

(3) three pieces of the same type.

Neither player will accept a piece selection if any of its pieces are of size less than $\epsilon$. If two or more piece selections are available in a given preference category, with all pieces greater than $\epsilon$ in size, the player chooses the option with the greatest total size (if two choices have the same total size, choose the lexicographic first option). Players only move to a lower ranked preference category if all options in a higher category contain a piece of cake with size less than $\epsilon$.

We show that for any set of pieces that player $A$ chooses, player $B$ prefers a piece selection which is not disjoint from $A$ 's. Suppose $A$ chooses three pieces of the same type; without loss of generality, say $A$ chooses $a a a$. Thus, each piece $a$ has size greater than $\epsilon$. If $B$ 's piece selection were disjoint from $a a a$, 
it would contain no $a$, and hence would contain at least two b's or two c's. However, replacing one of those repeated letters with an $a$ would result in a piece selection more desirable to $B$.

Next, suppose $A$ 's piece selection consists of two pieces of one type and one piece of another; for example, say $A$ chooses $a a b$. In the third cake, piece $a$ must have size less than $\epsilon$, otherwise $A$ would have chosen aaa. Therefore, to not conflict with $A$ 's choice, $B$ 's piece selection must contain piece $c$ in the last cake. Non-conflicting choices for $B$ are $b b c, b c c$ or $c b c$; however, the $a$ pieces in the first two cakes have size greater than $\epsilon$, so the piece selections $a b c$, bac or $a b c$ would be preferred by $B$ to the previous options, respectively. These conflict with $A$ 's piece selection in one of the first two cakes.

Finally, suppose $A$ chooses three different types of pieces, for example, $a b c$. Since $A$ would prefer $b b c$ or $c b c$ to $a b c$, it must be that $a$ is the only piece of size greater than $\epsilon$ in the first cake. Therefore $B$ will also choose piece $a$ from the first cake, and $A$ 's and B's piece selections will not be disjoint.

We have shown that a division with disjoint envy-free piece selections for two players need not exist for three cakes divided into three pieces each. It is easy to see that the same is true for four or more cakes. Simply have the players adopt the preferences described above on the first three cakes.

\subsection{Three cakes, four pieces each}

We now show that if, instead, the three cakes are each divided into four or more pieces, we can always find a division with envy-free piece selections for two players.

Theorem 8. Given 2 players and 3 cakes, there exists a division of the cakes into 4 (or more) pieces each that contains disjoint envy-free piece selections for both players.

As before, the idea of the proof is to use the Polytopal Sperner Lemma (Theorem 1) on the 9dimensional space of divisions $P$. However, our task is made much simpler by focusing on a particular full cell: the one that covers the center of $P$.

Proof. Let $P$ be the $(64,9)$-polytope in $\mathbb{R}^{12}$ of divisions of 3 cakes into 4 pieces each, and let $T$ be a triangulation of $P$ with a uniform owner labeling. By Theorem 5, the fully-labeled cells of $T$ cover $P$ under the map $f$. Hence, for at least one fully-labeled 9-simplex $\sigma, f(\sigma)$ contains the center of $P$. That is, there exist weights $a_{i}>0$, such that $Q=\sum_{i=1}^{10} a_{i} M_{i}$ where the $M_{i}$ are the matrices of the pure divisions corresponding to the vertices of $f(\sigma)$, and $Q$ is the $k \times m$ matrix in which every entry is $1 / 4$ (this is the center of $P$ ).

It will be convenient to visualize the vertices of $P$ arranged on a $4 \times 4 \times 4$ grid, since there are four pieces $\{a, b, c, d\}$ that may be selected for each of three cakes (see Fig. 7).

Note that there are four planes in each of the 3 orthogonal grid directions; in the rest of the proof, a plane will refer to one of these 12 special planes. For example, the bottom horizontal plane contains all vertices of $P$ corresponding to pure divisions in which piece $a$ is picked in the 3rd cake. In fact, any given plane corresponds to pure divisions in which a particular piece in a particular cake is chosen. The matrices corresponding to such divisions all have a 1 in the same entry. Since $Q$ is a weighted average of 0-1 matrices $M_{i}$ that represent pure divisions, and matrices with a 1 in a particular entry correspond to vertices in our grid that lie on a common plane, the total weight of vertices of $f(\sigma)$ that lie on a given plane of our grid must be $1 / 4$. All of our arguments will be based on this fact.

A vertex in our $4 \times 4 \times 4$ grid may also be thought of as a piece selection. By doing so, we see that two piece selections lie on a common plane if and only if they are not disjoint.

Recall that $\sigma$ denotes the full cell whose image $f(\sigma)$ contains the center of $P$. Consider a graph $G$ whose nodes are the labels of $\sigma$, and in which two nodes are adjacent in $G$ if and only if they represent disjoint piece selections, i.e., if and only if their labels do not lie on a common plane in Fig. 7.

We now show, in all arguments that follow, that $G$ has a connected component of size at least 6 . Since the uniform owner labeling of a 9-simplex has exactly 5 vertices owned by $A$ and 5 by $B$, this would imply the desired conclusion: there is an $A-B$ edge of $\sigma$ with disjoint preference labels.

Call a configuration of 4 vertices in the $4 \times 4 \times 4$ cube a diagonal if each plane contains exactly one vertex. These corresponding piece selections are all mutually disjoint, and so they form a size- 4 clique in $G$. Furthermore, any other vertex in the cube lies on exactly three planes, and so must be disjoint from one of the diagonal vertices. Thus, a set of vertices that contains a diagonal is connected in $G$. 


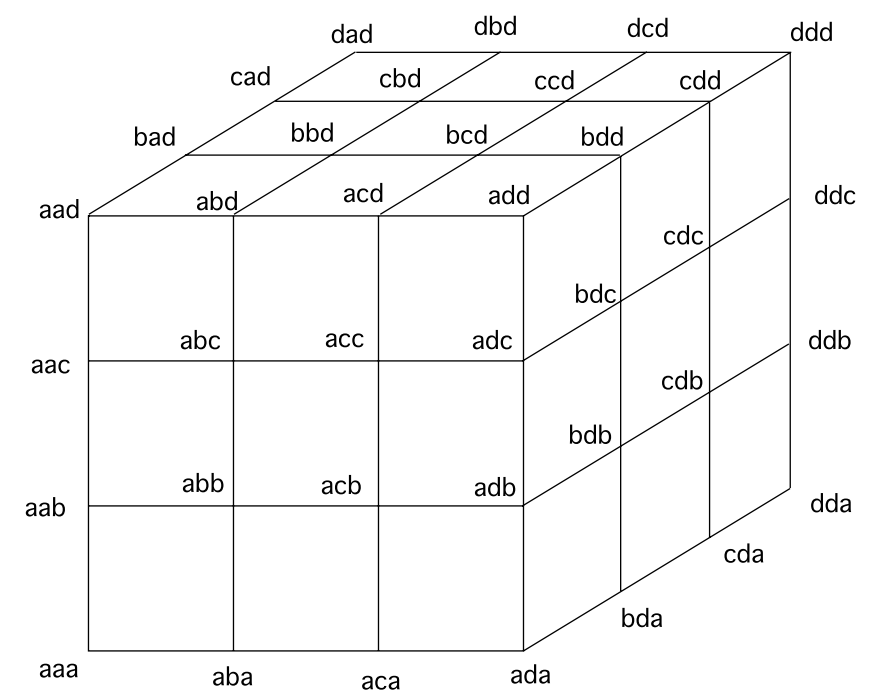

Fig. 7. The vertices of $P$, the pure divisions, arranged in a $4 \times 4 \times 4$ grid. These also may be thought of as piece selections, and two piece selections are disjoint if and only if they do not lie on the same grid plane.

We now focus attention on the number of vertices of $f(\sigma)$ on each plane of the cube and their associated weights $a_{i}$. Consider first the case in which some plane contains only one vertex, $v_{j}$; hence $a_{j}=1 / 4$. Vertex $v_{j}$ is adjacent, in $G$, to every other vertex with non-zero weight, since $v_{j}$ cannot lie on a plane with any other weighted vertex (otherwise the total weight of the plane exceeds $1 / 4$ ). If there are at least five other vertices with positive weight, $G$ would have a connected component of size at least 6 . Suppose there are five or fewer weighted vertices. Since the four planes in any given direction must each contain at least one weighted vertex, there must be three planes that contain exactly one weighted vertex. These vertices each have weight $1 / 4$, and each is the only vertex on any of the three orthogonal planes that contain it. Therefore, any additional weighted vertex must lie in the unique intersection of the remaining planes, and this in turn implies a diagonal configuration of vertices. By our earlier argument all 10 vertices of $\sigma$ are connected in $G$.

If there is no such plane, then every plane contains at least two vertices. We look at the possible cases individually and show that there is some vertex that lies on planes with at most 4 other vertices, and is therefore disjoint from at least 5 vertices in $G$ (resulting in a connected component of size at least 6 in $G)$. We will use the shorthand notation $(x, y, z, w)$ to indicate the number of vertices on the 4 planes in a given direction, ordered by the number of vertices they contain.

Case $1:(4,2,2,2),(4,2,2,2),(4,2,2,2)$. Suppose that in every direction there is one plane containing 4 vertices and three planes containing 2 vertices each. We claim that there exists a vertex which does not lie on any plane containing 4 vertices. To see why this is true, let the pairs of vertices on the three 2-vertex planes in one direction be $\{a, b\},\{c, d\}$, and $\{e, f\}$. The combined weight of each pair is $1 / 4$. If each of these vertices appeared on a 4-vertex plane in at least one of the other two directions, the sum of the weights of those two planes would be at least the combined weight of the six vertices $\{a, b, c, d, e, f\}$, or $3 / 4$, and so at least one of the planes would have weight greater than $1 / 4$, a contradiction. Therefore, there is a vertex which lies only on 2-vertex planes in each direction. This vertex is not disjoint from at most 3 other vertices, and so is disjoint from at least 6 .

Case 2: $(4,2,2,2),(4,2,2,2),(3,3,2,2)$. At most eight of the ten vertices of $\sigma$ lie on a 4 -vertex plane, so at least two do not lie on 4 -vertex planes. These have at most 4 neighbors in $G$ (it is four if they lie on planes with 2,2 , and 3 vertices). Thus, they are disjoint from at least 5 vertices in $G$.

Case $3:(4,2,2,2),(3,3,2,2),(3,3,2,2)$. By an argument similar to that of Case 1 , there exists a vertex which does not lie on the 4-vertex plane, and lies on at most one 3-vertex plane; otherwise, the two 3-vertex planes in each direction must contain all 6 of the vertices not on the 4-vertex plane - so the total weight of the two 3-vertex planes (in either direction) would be $3 / 4$, which is too large. 
Therefore, there exists a vertex that lies on at most one 3-vertex plane and no 4-vertex plane. This vertex has at most 4 neighbors in $G$ (it is four if it lies on planes with 2, 2, and 3 vertices). Thus it is disjoint in $G$ from at least 5 vertices.

Case $4:(3,3,2,2),(3,3,2,2),(3,3,2,2)$. There are twelve positions for vertices on 2 -vertex planes and only 10 vertices of $\sigma$, so by the pigeonhole principle, one vertex must lie on at least two 2-vertex planes, hence it has at most 4 neighbors in $G$, so it is disjoint in $G$ from at least 5 vertices.

Thus, given a fully-labeled 9-simplex $\sigma$ whose image under $f$ contains the point $Q$, a uniform owner labeling produces an $A-B$ edge with disjoint preference labels. By using the methods of Section 3 we are able to find a division with disjoint envy-free piece selections for players $A$ and $B$.

As in the proof of Theorem 6 , if the cakes are cut into more than 4 pieces each, we can guarantee an envy-free allocation by restricting our attention to the case in which the extra pieces are empty. Other envy-free allocations may exist as well.

\section{Discussion and open questions}

In this article, the Polytopal Sperner Lemma has given us insight into how many pieces are necessary for envy-free multiple-cake division with disjoint piece selections. We have shown that it is possible to divide two (respectively, three) cakes among two players in an envy-free fashion with disjoint piece selections so long as each cake is cut into at least three (respectively, four) pieces. This suggests a natural question for 2 players and $m$ cakes: is it always possible to find disjoint envy-free piece selections if each cake is cut into at least $m+1$ pieces? And can we get by with fewer pieces in some of the cakes (as we did for 2 cakes, allowing one cake to have 2 instead of 3 pieces)?

We have also shown that there may not exist envy-free divisions of two (respectively, three) cakes among two players when the cakes are only cut into two (respectively, three) pieces each. In fact, with preferences similar to those in the proof of Theorem 7 , one can verify that 4 cakes cut in 4 pieces each is not sufficient to ensure an envy-free allocation among two players. In this case, A's preferences in order are: 4 of a kind, 3 of a kind, 2 pair, 1 pair, all different. B's preferences are the reverse. By extending these preferences to $m$ cakes each cut into $m$ pieces, is it possible to show that disjoint envy-free piece selections may not exist for two players in this situation?

Finally, how many additional pieces do we need in each cake if there are more players who demand disjoint envy-free piece selections? Recall (see the comments at the end of Section 3) that disjoint, envy-free piece selections would by nature yield Pareto-optimal allocations, even with more than two players. Our intuition suggests that if we cut the cake into enough pieces, we should be able to satisfy all players, though there may be many extra pieces left over. We conclude with this open question: for given numbers of players and cakes, we ask for the minimum number of pieces to divide each cake that would ensure that some division has disjoint envy-free piece selections.

\section{References}

Brams, S.J., Edelman, P.H., Fishburn, P.C., 2001. Paradoxes of fair division. J. Phil. 98 (6), 300-314.

Brams, S.J., Taylor, A.D., 1996. Fair Division: From Cake-Cutting to Dispute Resolution. Cambridge University Press, Cambridge. Brams, S.J., Taylor, A.D., 1995. An envy-free cake division protocol. Amer. Math. Monthly 102, 9-18.

De Loera, J.A., Peterson, E., Su, F.E., 2002. A polytopal generalization of Sperner's Lemma. J. Combin. Theory Ser. A $100,1-26$.

Neyman, J., 1946. Un théorème d'existence. Comptes Rendus Mathématique Académie des Sciences Paris 222, $843-845$.

Robertson, J.M., Webb, W.A., 1998. Cake-Cutting Algorithms: Be Fair If You Can. A K Peters Ltd., Natick, Mass.

Simmons, F., Su, F.E., 2003. Consensus-halving via theorems of Borsuk-Ulam and Tucker. Math. Social Sci. 45, 15-25.

Sperner, E., 1928. Neuer Beweis für die Invarianz der Dimensionszahl und des Gebietes. Abh. Math. Sem. Univ. Hamburg 6, $265-272$.

Steinhaus, H., 1948. The problem of fair division. Econometrica 16, 101-104.

Su, F.E., 1999. Rental harmony: Sperner's lemma in fair division. Amer. Math. Monthly 106, 930-942.

Todd, M.J., 1976. The Computation of Fixed Points and Applications. In: Lecture Notes in Economic and Mathematical Systems, Springer-Verlag, New York.

Yang, Z., 1999. Computing Equilibria and Fixed Points. Kluwer Academic Publishers, Boston. 